\title{
LA MAQUINARIA SOCIAL Y LAS CIENCIAS HUMANAS. PENSIONADOS DE LA JUNTA PARA LA AMPLIACIÓN DE ESTUDIOS EN LA EUROPA DE LA RAZÓN TECNOCRÁTICA
}

\author{
Víctor Guijarro Mora \\ Universidad Rey Juan Carlos \\ victor.guijarro@urjc.es
}

Recibido: 18 junio 2014; Aceptado: 3 septiembre 2014.

Cómo citar este artículo/Citation: Guijarro Mora, Víctor (2015), "La maquinaria social y las ciencias humanas. Pensionados de la Junta para la Ampliación de Estudios en la Europa de la razón tecnocrática", Asclepio 67 (1): p086. doi: http://dx.doi.org/10.3989/ asclepio.2015.12

RESUMEN: La creación de la JAE y su política de pensiones promovió no solo los elementos integrantes del discurso oficial, sino otros intereses ligados a los enfoques tecnocráticos de los problemas sociales difundidos en la Europa de la modernidad. En este estudio exploramos este segundo efecto, no reconocido suficientemente, mediante el examen de la trayectoria y propuestas de diversos pensionados que viajaron entre 1909 y 1932 a Alemania, Inglaterra, Bélgica, Suiza y Francia.

PALABRAS CLAVE: Junta para la Ampliación de Estudios; Pensionados; Ciencias Humanas; Tecnocracia; Problemas sociales.

\section{SOCIAL MACHINERY AND THE HUMAN SCIENCES. THE JUNTA PARA LA AMPLIACIÓN DE ESTUDIOS SCHOLARSHIPS IN THE TECHNOCRATIC EUROPE}

ABSTRACT: The establishment of the JAE and the scholarship policy supported by this institution promoted not only the well-known scientific renovation, but other values related in this case to the technocratic views of social problems. The present study is aimed at exploring this second effect, a relatively unattended topic, by the examination of the concerns and purposes of various scholarship holders who traveled between 1909 and 1932 to Germany, England, Belgium, Switzerland, and France.

KEY WORDS: Junta para la Ampliación de Estudios; Scholarships; Human Sciences; Technocracy; Social Problems. 
El establecimiento de la Junta para la Ampliación de Estudios en 1907 sirvió, como se ha afirmado, para activar un programa de renovación científica que, según el ideario de Cajal, debía contribuir a la revitalización de la cultura española ${ }^{1}$. Uno de los instrumentos de este plan fue la política de pensiones, cuyo propósito era poner en contacto los científicos españoles con colegas extranjeros y con laboratorios donde se empleaban los procedimientos de investigación más avanzados. Pero junto estos ideales se promovieron otros que tenían que ver con la extensión de los enfoques tecnocráticos ${ }^{2}$ a las ciencias humanas y a la resolución de problemas sociales y políticos acuciantes. Profesionales interesados en la educación, la orientación profesional y la planificación urbana, seducidos por la autoridad de las tecnociencias, pretendieron ofrecer soluciones que preservaran el orden social y evitaran el desarraigo de las multitudes, unas propuestas que se distanciaban tanto del crudo taylorismo como de las actitudes revolucionarias o de los elitismos displicentes. Como mantenía el alemán Hugo Münsterberg, uno de los iniciadores de la psicotecnia en Europa y Norteamérica, se buscaba un campo de estudios neutral alejado tanto del capitalismo despiadado como de las posiciones sentimentalistas (Münsterberg, 1913, pp. 5-11 y 18-19).

Eran unas propuestas que debían asegurar la adaptabilidad de amplios colectivos a los marcos institucionales y económicos y que, según se pensaba, debían mejorar, al mismo tiempo, la percepción que de sí mismos tenían los nuevos sujetos sociales, habitualmente excluidos de un sistema que los ignoraba. Tests, pruebas mecanizadas, aparatos normalizados, nuevas orientaciones pedagógicas y planificación urbana fueron algunos de los recursos ensayados para conseguir los propósitos mencionados ${ }^{3}$. Procedimientos y rutinas que fueron asimilados por algunos profesionales españoles vinculados de una u otra manera a la formación técnica, cuyos intereses se vieron favorecidos por la política de pensiones de la JAE. En este estudio seleccionamos una muestra de esos pensionados que en sus viajes ${ }^{4}$ a centros emblemáticos, como la Escuela de Decroly, la Escuela Normal Superior de Saint Cloud, la Universidad Industrial de Charleroi o el Instituto de Psicología Experimental de Leipzig, comprobaron la relevancia otorgada a las técnicas científicas tanto para el estudio de las capacidades mecánicas e intelectuales de los individuos como para conseguir el ajuste social de amplios colectivos a los nuevos modelos productivos, profesionales, de entretenimiento y de gestión industrial.

Como afirma Charles L. Sanford, "La principal cuestión cultural subyacente del periodo no era el hecho de si el hombre occidental pertenecía a la máquina, sino en qué condiciones" (Sanford, 1981, p. 806). Algunos pensadores y referentes de la inteligencia y el arte europeo de las primeras décadas del siglo XX habían cuestionado la relación de subordinación existente entre los sujetos y las máquinas, ya fuera por el sometimiento al ritmo de la producción industrial o por la admiración que provocaban (Irigoyen, 2002). Uno de esos intelectuales, y también pensionado, fue Ortega y Gasset. Cuando el filósofo español empleó la expresión "Europa es ciencia" en el discurso pronunciado en la Asociación para el Progreso de las Ciencias el año de su fundación (1908), quería decir que los logros técnicos y avances que observamos derivaban del cultivo del conocimiento científico (Ortega, 1908, p. 3). Esta insistencia en la subordinación de la tecnología a la ciencia, tiene como objeto, como se pone de manifiesto en alguno de los trabajos reunidos en Meditaciones sobre la técnica (1935), la relatividad de las labores dedicadas a la producción artefactual (Ortega, 2006, pp. 421-427). Cuando relacionamos estas afirmaciones con las realizadas con anterioridad en La rebelión de las masas (1930) se desprende que en Ortega pervive una visión intelectualista y elitista que alienta un acusado desdén por el ejercicio técnico y especialmente por su poder de seducción (Ortega, 2006, pp. 564-567) . $^{5}$. La técnica, entendida como un fenómeno propio de una época y como suma de ciencia e industria, era para el filósofo español una de las razones de la sociedad masificada (Esquirol, 2001, p. 21).

Pero no todos los pensionados compartían este concepto de modernidad. Los que examinamos en este trabajo, en cambio, se plantearon las "condiciones" de la pertenencia de los sujetos a las máquinas sin cuestionarse su dependencia o no de los artefactos. Así, en el primer apartado llamamos la atención sobre algunos casos, como el de Lorenzo Miralles, Antonio Ballesteros y Luis Valerí, enmarcados en el mundo de la pedagogía técnica, que transmiten en las impresiones que reúnen en sus viajes su interés por las manifestaciones de la cultura tecnológica e industrial y, dentro de este marco, por los valores que se derivan de los programas educativos recreados en los centros visitados. Como se verá, los pensionados destacan que ya en la escuela primaria se observa un interés por acercar el pensamiento a la acción y, en general, por poner en práctica actitudes antiintelectualistas, propósitos que consiguen dignificar las labores manuales y prácticas, tradicionalmente excluidas de las apreciaciones clasistas.

En el segundo apartado, nos ocupamos de diversos ejemplos relativos a la psicología aplicada y a la psicotecnia, disciplinas cuya finalidad era realizar aportaciones a la integración de las personas a los marcos sociolaborales y a la resolución de la conflictividad social atendiendo a procedimientos científicos. Aquí se siguieron dos estrategias: la conversión de la pedagogía en una ciencia, mediante el 
examen en las fases tempranas del aprendizaje de las capacidades intelectuales y motrices, y la centrada en la orientación profesional, en la búsqueda de la ocupación idónea en función de las competencias de los individuos. Como se mostrará, recibieron una influencia de esta visión basada en los modelos de la psicología experimental varios pensionados, entre ellos Eloy Luis André, José del Peso y Salvador Ferrer.

En el tercer apartado, por último, examinamos los casos de Rafael Campalans y Julián Besteiro en relación con la promoción de una estética utilitarista al servicio del planeamiento urbano y del acceso de las clases desfavorecidas a las viviendas y a los entornos a escala humana, siguiendo para ello los presupuestos de un ordenamiento racionalista de la ciudad.

En estas tres dimensiones, la educativa, la profesional y la residencial se observa la presencia arquetípica de modelos que enaltecen la eficiencia funcional tecnocrática como respuesta al creciente protagonismo de sujetos sociales animados por la expansión de la civilización industrial en Europa, Japón y Estados Unidos. Unas posiciones que significaban una alternativa al cambio basado en la acción revolucionaria de la clase obrera o desarraigada y que se aproximaban, en cambio, a las actitudes moderadas y reformistas del socialismo y el liberalismo ya extendidas desde finales del siglo XIX y principios del XX. A continuación, siguiendo la división establecida, ampliaremos estas indicaciones con detalles procedentes de los efectos, expresados en memorias y proyectos, que los desplazamientos a establecimientos de Berlín, Leipzig, Londres, París, Bruselas y Ginebra tuvieron en los pensionados.

\section{LA FORMACIÓN TÉCNICA Y MORAL DEL NUEVO OBRERO}

Desde la llustración, la educación técnica tenía dos propósitos: el economicista, centrado en el aprendizaje de instrumentos para rentabilizar los recursos naturales, y el moral, cuyo cometido era conseguir la integración social del individuo e incrementar su autoestima a través de la formación práctica y la atención a las habilidades (Pannabecker, 1996). El segundo objetivo subrayaba la importancia de la técnica como elemento esencial del desarrollo humano. Comprobaremos que en las pensiones concedidas por la Junta para la Ampliación de Estudios se canalizaron y promovieron en el campo de la pedagogía visiones particulares enmarcadas tanto en la retórica del beneficio nacional como en la de la regeneración de personas cuya vía para ser eficaz en los modelos de la economía productiva dependía básicamente de su capacitación práctica.
Teresa Marín, en La renovación pedagógica en España, afirma que en el sector de la educación se observan diversos periodos en los que hay un incremento apreciable en la concesión de pensiones (Marín, 1999, pp. 120-128). Uno, el que va de 1910-1912; otro, desde 1920-1922 y, un tercero, el comprendido en el bienio 1924-25. El primero se explica por diversas causas, entre ellas las medidas sobre educación de adultos y la implantación en España de la Escuela del Hogar y Profesional de la Mujer, que influyó en el aumento de demandas a la Junta sobre estos temas. El segundo está relacionado con la creación de los Institutos-Escuela y del Grupo Escolar Cervantes en Madrid. El tercero nos interesa particularmente por su relación con la enseñanza técnica. La causa que se señala para explicar la subida es la promulgación en 1924 del Estatuto de Enseñanza Industrial (Decretoley de 31 de octubre). Este, según se apunta en el texto, regulaba la formación del obrero, que presentaba diversas peculiaridades y que no era reducible a la enseñanza regular proporcionada en el sistema de educación primaria o superior. Además, estos hechos coincidían con la presentación en esos años del proyecto socialista de educación, en el que junto a otros cometidos se pretendía impulsar la enseñanza profesional y dignificar las actividades no intelectuales mediante la atención a los trabajos manuales. Además del grupo beneficiados por las pensiones según las razones aportadas por Marín Eced, en el primer periodo encontramos a Lorenzo Miralles, profesor de una escuela de artes y oficios e interesado por aplicar en los niveles educativos primarios reformas que vincularan la enseñanza técnica con las demandas sociales ${ }^{6}$. Este interés de personas con formación científica o técnica o bien con intereses en la educación práctica que iban más allá de la estricta aplicación profesional, aparecerá posteriormente reproducido, como se verá, en la tercera etapa, dentro del marco político ya aludido.

El mencionado Lorenzo Miralles era catedrático de Aritmética y Geometría en la Escuela Superior de Artes e Industrias de Almería cuando recibió la pensión para viajar en 1909 y 1910 a Francia, Bélgica e Inglaterra. En la memoria que elaboró posteriormente aparecen algunos presupuestos que consideramos de interés. Aseguraba allí que la ciencia no solo era imprescindible para la organización de la industria, la agricultura, la higiene, sino para otros cometidos como la "formación del espíritu", ideas que están activas solo en algunos centros de manera excepcional, como en la escuela de Decroly (Bruselas) y en el Bedales School (Petersfield, Inglaterra) (Miralles, 1911, p. 150). En Francia, asegura, se ocupó de las líneas generales del plan para la enseñanza primaria, así como de la educación de los profesionales, donde destaca la formación científica y técnica que reciben sus estudiantes, y en particular la atención que se dispensa 
a los trabajos manuales. Describe después algunos detalles sobre cómo tienen lugar las "lecciones de cosas": los temas que se tratan, qué cuestiones plantea el docente y los materiales que se emplean. Para Miralles estas prácticas representan una forma de introducir las ciencias experimentales, unas materias que comprenden, teniendo en cuenta el plan completo de enseñanza elemental, además de diversas nociones elementales, la transformación de materias primas en elaboradas (curso elemental), agricultura (curso medio, 9 a 11 años, y superior, 11 a 12). Añade que la agricultura se imparte en escuelas ubicadas en distritos rurales y que él mismo visitó una en Cheville provista de un jardín para el ensayo de abonos y la realización de prácticas (Miralles, 1911, pp. 159-169). "En íntima relación con la enseñanza de las ciencias experimentales -afirma-, debemos poner el cultivo de la habilidad manual. El alumno en la escuela dibuja, modela y maneja los útiles de carpintería; así que, al salir de ella, lleva materiales bastantes o para continuar sus estudios o para ingresar en la industria si es esta su inclinación" (Miralles, 1911, p. 160).

Pero los procedimientos aplicados en la primera etapa, que refuerzan la capacidad de observación y experimentación individual y autónoma, se abandonan poco a poco hasta perderse en los niveles superiores. La enseñanza superior presenta así diversas deficiencias. Cuando visitó Bélgica la impresión fue diferente. Sus escuelas experimentales proporcionaban una educación tanto general como técnica. Elogia el sistema de enseñanza en el que las lecciones de cosas pasan a llamarse lecciones de observaciones y donde se fomenta el aprendizaje individual, la experimentación, la importancia del trabajo manual y las visitas a museos, fábricas y talleres. Menciona como teóricos de referencia a Ovide Decroly, Jean Demoor, Alexis Sluys y el "utilitarismo científico" de H. Spencer (Miralles, 1911, p. 169). Este último se invoca en los procedimientos que se aplican especialmente en la Escuela de San Gil, lugar en el que para proporcionar una "educación manual integral" al futuro obrero se considera improcedente todo aquello que distraiga del interés inmediato, como las puras teorías, abstracciones, teoremas, reglas, etc. (Miralles, 1911, pp. 169-170) Particularmente le llama la atención el protagonismo que en la enseñanza tiene el "sentido muscular". Cita aquí una conferencia de Decroly, en la que se insiste en la importancia de ejercitar el tacto, la habilidad manual, que es "asiento de muchos sentidos" y vía fundamental para la "educación y desarrollo del cerebro" (Miralles, 1911, p. 171).

Análogas sensaciones de admiración le produjo su visita a Inglaterra, el país en cuyas manos estaba, según pensaba, el destino del mundo. Una percepción que debía tener un reflejo en el sistema educativo. Aunque sus impresiones no le defraudaron, el sím- bolo supremo del acierto en la formación primaria seguía siendo la Escuela de Decroly de Bruselas ${ }^{7}$. La razón es que era el único lugar donde lo principal no era la lectura, escritura y las cuentas, como en la mayoría de los centros visitados, sino la observación de la naturaleza, los juegos, los ejercicios corporales y los trabajos manuales (el dibujo, el modelado, la jardinería, la carpintería, etc.) (Miralles, 1911, pp. 197-198). La insistencia tanto aquí como a lo largo del texto en la observación y la experiencia directa como base insustituible del aprendizaje significaba que el conocimiento y, en particular el conocimiento técnico, era el resultado de un proceso de prácticas y ensayos personales. Era pues posible una tecnología que se originara en los propósitos individuales y en sus inclinaciones naturales, no en los grandes proyectos. Aún se manifiestan aquí los propósitos vinculados a la tradición de las artes y oficios, defendidos en el siglo XIX en contra de la pura producción estandarizada por pensadores como John Ruskin o William Morris. Una visión que veremos reflejada en parte en el ideario que se asoció al desarrollo de los Institutos-Escuela españoles, ensayos de una nueva pedagogía que comenzaron su andadura en $1918^{\circ}$. Pero también constatamos un factor que se convertirá en una constante durante el periodo que estudiamos: el antiintelectualismo como forma de reducir las diferencias clasistas y dignificar las tareas manuales.

En los años 1924 y 1925 hubo un ambiente favorable a la educación técnica, y la Junta fue sensible a esas prioridades. Comenta Teresa Marín que "La industrialización y los movimientos obreros -sobre todo la influencia del pensamiento marxista- habían creado en Europa escuelas técnicas de todo tipo. La educación del obrero era uno de los objetivos a alcanzar. De ahí que la formación profesional, el nuevo concepto de instrucción como factor de producción, la socialización de la cultura, las relaciones escuela-trabajo, etc., influyeran en el profesorado a la hora de solicitar sus pensiones y, en la Junta, a la hora de concederlas" (Marín, 1999, p. 128). Pero también cabe añadir otras consideraciones, en este caso señaladas por Álvaro Soto Carmona, que tienen relación con un acontecimiento ya mencionado, la promulgación en 1924 del Estatuto de Enseñanza Industrial. El historiador indica que el Estatuto aprobado durante la Dictadura de Primo de Rivera fue la respuesta oficial a los cambios que se estaban produciendo en el sistema de producción, cada vez más condicionado por la lógica de la competencia, de la productividad, de la especialización y del maquinismo. En este contexto se planteó como imprescindible la mejora de los procedimientos educativos, cuya finalidad era contar con trabajadores que se adaptasen a los cambios técnicos y a las nuevas tareas que surgían al aplicar la filosofía de la división del trabajo en las instalaciones industriales (Soto, 1989, pp. 207-244). Probablemente también 
había que transmitir al trabajador una disciplina, que se asimilaba en el proceso de alfabetización general y de alfabetización tecnológica iniciado en las escuelas primarias y especiales. Para Álvaro Soto, el propósito del Estatuto según los argumentos ofrecidos era reorganizar las enseñanzas industriales para acomodarlas a la nueva economía e igualmente evitar el desorden que las iniciativas locales y provinciales provocaban, en clara alusión a los proyectos de la Mancomunitat catalana. Como parte de esta reforma se pretendía facilitar el aprendizaje de un oficio y ofrecer medios para que estos servicios llegaran al mayor número de obreros (Soto, 1989, p. 226). También se preveía la creación de institutos de orientación profesional, a los que nos referiremos más adelante. Pero la ineficacia del decreto en la práctica fue uno de los motivos para que el 21 de diciembre de 1928 se promulgara el Estatuto de Formación Profesional. La aplicación del nuevo Decreto permitió el incremento de las escuelas industriales; así el número medio de habitantes por Escuela de Trabajo bajó de 1.097.954 en 1926 a 238.909 en 1930 (Soto, 1989, p. 228).

Algunos beneficiados por la concesión de pensiones en los años citados fueron: José del Peso Sevillano, Rodolfo Tomás Samper ${ }^{9}$, Luis Castellá y Lloverás, Gervasio Manrique Hernández, Leonor Serrano Pablo ${ }^{10}$, Luis Valerí Sahis y Antonio Ballesteros Usano. Las ideas y grandes propósitos de los interesados en la renovación de la pedagógica técnica no difieren en una medida significativa de lo ya expuesto al hablar de Miralles. Encontramos así que la mayoría seguía sintiéndose atraída por las prácticas docentes de Francia, Bélgica y Suiza. El idioma era sin duda uno de los factores determinantes para esas elecciones, pero también seguían influyendo las expectativas de encontrar un modelo alternativo y prometedor que suplantara el sistema español. Los profesores e inspectores encontraron esas variantes ajustadas a sus preferencias en parajes atractivos. Por ejemplo, en el extrarradio de París, a una hora del centro en vapor desde PontRoyal, se encontraba la Escuela Normal Superior de Saint Cloud. Lo más destacado de la institución era el carácter práctico de sus enseñanzas, la relevancia concedida a las "ciencias aplicadas" y la insistencia en la metodología activa:

los becados españoles que visitaron esta Escuela, ponderaron repetidas veces el antiintelectualismo y el sentido práctico de las enseñanzas que se impartían en Saint Cloud: Los gabinetes de Física, Química y Ciencias Naturales, en los que cada alumno tenía su mesa, su colección de frascos con los principales reactivos y su instrumental de experiencias (probetas, tubos de ensayo, matraces, tijeras, mecheros...), amén de los aparatos más modernos en una y otra ciencia, despertaban las más entusiastas alabanzas en propios y extraños (Marín, 1988, pp. 97-98).
Por su parte, de Bélgica, con un sistema descentralizado, debieron llamar la atención a quienes les preocupaban los entresijos de la formación profesional las escuelas-taller, donde la enseñanza se articulaba en torno al dibujo, los trabajos manuales y el preaprendizaje de un oficio. Antonio Ballesteros, Inspector de Primera Enseñanza y favorecido con tres pensiones (1921, 1924-25 y 1936), escribió en la memoria redactada tras el segundo viaje palabras de admiración hacia los métodos belgas, donde destaca de nuevo la atención a la experiencia y la preparación profesional que recibe el estudiante:

$Y$ de este modo, por la labor que la escuela realiza puede presentarse como modelo de habilidad y de inteligencia al obrero belga, así Bélgica tiene sus campos perfectamente cultivados, dando la Agricultura un máximo rendimiento y llega en su industria a esa perfección y esa esplendorosa prosperidad que coloca a esta nación ejemplar a la cabeza de los pueblos industriales del mundo (Ballesteros, 1925, p. 231).

La formación científico-técnica ya no solo respondía a las inclinaciones naturales y a la natural evolución del individuo, o bien atendía a su formación espiritual-integral, sino que ahora estaba vinculada de igual manera a las demandas productivas y prioridades estatales, según se reconoce en el texto anterior.

La pensión de Luis Valerí Sahis ${ }^{11}$, concedida en 1925 para una estancia de tres meses en Francia, Bélgica y Suiza, estuvo vinculada a la promulgación del Estatuto citado ${ }^{12}$. Así lo declara en el extenso documento de su solicitud, donde reproduce los ideales de la enseñanza técnica, presentes en los proyectos de minorías e instituciones destinados a cambiar la condición de las clases menos favorecidas en los siglos XIX y XX. Comenta allí que "van a establecerse en España nuevos organismos de enseñanza, cuya transcendencia tanto en el orden social de mejoramiento de la clase obrera, como en el orden técnico de perfeccionamiento industrial es, a todas luces, de extraordinaria importancia"13 y, a continuación, recuerda los valores que acompañan a este aprendizaje: el estudiante recibirá "al mismo tiempo que una instrucción técnica general al principio y especializada más tarde [ ] una educación moral que eleve su condición, inculcándole el principio del alto valor espiritual del trabajo"14. Celebraba en su escrito que entre las autoridades gubernamentales se hubiera tomado conciencia de la nueva realidad que representaba el obrero industrial. Su formación demandaba, según asegura, un nuevo tipo de metodología donde la atención a la experiencia fuera el factor más relevante. El cambio que defendía no atendía solo a una dimensión, sino que, según las áreas de interés que menciona en el plan de su viaje, afectaba a una variedad de parcelas que rodeaban 
esas prácticas, como las relaciones escuela-industria, la organización de las cámaras de comercio, los sindicatos de obreros y los aspectos legislativos que afectaban a la regulación local del aprendizaje. No solo se buscaba pues información sobre aspectos técnicos, sino también sobre los jurídicos y los sociales, sin los cuales posiblemente los primeros no se podrían atender adecuadamente. Prestó atención especialmente al método francés, tarea que se llevó a cabo mediante entrevistas con patronos, obreros y personal perteneciente a sindicatos y cámaras (JAE, 1927, pp. 121-124). Cuando ofrece detalles de su itinerario, no faltan, como en otras solicitudes, la mención a alguna institución emblemática, cuyo prestigio como un argumento más para reforzar los motivos del viaje. Así en Bélgica destaca "la gran Universidad Industrial de Charleroi, monumento de sabiduría e institución ejemplar en todos los órdenes de la enseñanza profesional obrera" (JAE, 1927, p. 124).

\section{LA ADAPTACIÓN AL NUEVO ORDEN SOCIOTÉCNICO}

Las ciencias humanas, según se demandaba, debían ofrecer una respuesta a los cambios que estaban teniendo lugar en el sistema de producción, distribución, venta y selección de personal, cambios que desde 1900 afectaron a la concepción de la gestión ("management") empresarial e industrial. Uno de los primeros intentos de esta aproximación científica fue el taylorismo, que tomó sin embargo como patrón de eficiencia la ingeniería y las nociones de mecánica ${ }^{15}$. El prestigio de la tecnología, comprobado a través de los inventos que transformaban el mundo doméstico y el paisaje urbano, ofrecía ejemplos para confiar en los planteamientos anteriores. Pero la crudeza de sus principios provocó un rechazo apreciable en medios artísticos e intelectuales, oposición que fue inmortalizada en dos clásicos de la historia del cine: Metrópolis, de Fritz Lang (1926), y Tiempos modernos, de Charlie Chaplin (1936). En los círculos académicos se pensó que la vía más adecuada para tratar la adaptabilidad de los sujetos a los entornos mercadotécnicos era el examen psicológico, lo que intensificó el interés por las estimaciones centradas en diversas parcelas del sujeto, como la respuesta motora, la percepción, el aprendizaje y, sobre todo, la clase de estímulo que podía aumentar o disminuir la fatiga. Pero comprobaremos que para explorar la adaptabilidad del ser humano a los entornos artificiales, esta alternativa no se desprendió de los modelos e imágenes mecánicas, procedentes de la tradición de la fisiología, la psicología experimental y la antropometría, con una clara tendencia a la disociación del sujeto.

También buscaron los pensionados en Europa evidencias para convertir a la pedagogía en una disciplina verdaderamente científica, rigurosa y precisa, y para ello mostraron una elevada curiosidad por las técni- cas producidas en el seno de los laboratorios y los gabinetes (Sorbona, El Colegio de Francia, Universidad Libre de Bruselas, Leipzig ). Igualmente aprendieron a usar los tests y los procedimientos psicotécnicos y psicométricos de la psicología experimental, con los que esperaban conocer con precisión las aptitudes y capacidades de los estudiantes y efectuar acertados procesos de selección de profesionales. Esto significaba trasladar a la población procedimientos de autocontrol basados en las rutinas sociotécnicas.

En España llamamos la atención, primero, sobre dos personajes: Eloy Luis André, por su interés, en principio llamativo, por introducir la filosofía de la orientación ocupacional en los institutos, y Emilio Mira y López (1896-1964), quien si bien no puede considerarse propiamente un pensionado de la JAE (la solicitó pero no fue aceptada), sí fue comisionado en 1921 para visitar centros de orientación profesional y de psicología experimental europeos. Mira representa un buen ejemplo de los esfuerzos por convencer a una población escéptica de las bondades de los procedimientos tecnocientíficos y de su aplicación al asesoramiento laboral. Es decir, comparte intereses con otros expertos españoles de la psicología aplicada, además de haber colaborado con el grupo de Madrid en la elaboración del mencionado Estatuto de Formación Profesional. Después, nos ocuparemos de otros pensionados que atendiendo a problemas similares concibieron proyectos de investigación propios.

El primero fue pensionado en 1909 por la JAE y fue catedrático de Psicología, Lógica, Ética y Rudimentos de Derecho en el Instituto Cardenal Cisneros de Madrid desde $1919^{16}$. Nunca profesó simpatías hacia los movimientos del krausismo positivista ni de los conservadores neoescolásticos. En "El porvenir de la Filosofía científica en España e Hispano-América" explica los motivos de su viaje a Alemania, así como sus discrepancias con el fervor europeizante de los institucionistas (André, 1912). Allí medita sobre la promoción de una especulación filosófica que tenga en cuenta los resultados de las investigaciones científicas. Y añade: "Aquí, a Alemania, hemos venido con el propósito de buscar instrumentos e iteración para el propio trabajo en nuestro hogar y solar espiritual. Esta ayuda y este apoyo no nos despersonaliza de ningún modo [] Nosotros, más modestos en nuestra labor, queremos indicar con esto, que el despertar a la vida espiritual de un pueblo, solo se logra haciendo percutir en su ámbito mental los fuertes aldabonazos y el poderoso murmullo del tráfago científico de otro, que le sirva de mentor". Los "instrumentos" y el espíritu científico modélico al que se refiere tanto aquí como en su solicitud ${ }^{17}$ los había encontrado en la universidad de Leipzig, donde André asistió a cursos y seminarios y trabajó en el Instituto de Psicología Experimental junto a William Wundt (Müllberger, 2014). Escribió 
entre otras obras un manual de psicología experimental, cuya cuarta edición incluye una parte dedicada a la psicología individual, donde se tratan cuestiones de psicología ocupacional. De análogo interés es la colección de instrumentos perteneciente al laboratorio de psicología del instituto madrileño, donde trabajó André y que en la actualidad se conserva parcialmente (Rodríguez, 2012). Conforman una muestra que remite a la tecnología de los recursos corporales y mentales, una tecnología diseñada para medir la inteligencia y las aptitudes técnico-prácticas. El interés de los planteamientos de André consiste en que si bien por sus concepciones sustancialistas del alma no sigue los presupuestos de la ortodoxia wundtiana ni del positivismo estricto, introdujo en su Psicología experimental, manual empleado en sus lecciones, consideraciones teóricas relativas al estudio de la fatiga, uno de los conceptos básicos de los análisis sobre la adaptabilidad persona-máquina y de las comprobaciones acerca del rendimiento de los trabajadores en el espacio de la factoría (André, 1931, pp. 318-320) ${ }^{18}$.

Por otra parte, Emilio Mira, quien mantuvo un espíritu pragmático que valoraba la vertiente práctica y no la especulativa de la ciencia, llegó a ser catedrático de Psiquiatría de la Universidad de Barcelona en 1933. Después de constituida la Mancomunitat, el Museo Social creado en 1913 (y dirigido por un ingeniero industrial, J. M. Tallada) fue transformado en el Secretariado de Aprendizaje, destinado a modernizar las funciones del anterior organismo ${ }^{19}$. Este dio lugar a dos instituciones: la Escuela de Trabajo o Universidad Industrial, cuyo cometido era la formación profesional y humana de aprendices y que dirigió Rafael Campalans $^{20}$, y el Instituto de Orientación Profesional (IOP), donde entre otras secciones contaba con un laboratorio de psicofisiología, dirigido por Emilio Mira, y otro de antropometría cuyo jefe era Luis Trías de Bes. En 1921, con motivo de la organización de la Segunda Conferencia Internacional de Psicotecnia ${ }^{21}$, es comisionado por el IOP para visitar los centros europeos más destacados de orientación profesional y de psicología experimental, como el laboratorio de Lahy, en París; de Decroly, en Bruselas; de Myers, en Londres; de Claparède, en Ginebra; de Lippmann, en Múnich; de Moede, en Berlín y de Ferrari, en Milán (Iruela, 1993, p. 61).

Para difundir la existencia del IOP se llevó a cabo una campaña dirigida a la población en general y a las escuelas, en particular, en la que se informaba sobre sus servicios. Se ofrecía asesoramiento gratuito a los jóvenes para que eligieran la profesión más adecuada a sus capacidades. Cuando llegaban se les abría una ficha y se tomaba nota de sus características generales. Después se procedía a la realización de pruebas en el Laboratorio Médico-Antropométrico, donde se determinaban sus aptitudes físicas, y también en el Labo- ratorio Psicométrico, donde se establecían sus aptitudes mentales. Con los datos se informaba al individuo examinado sobre las competencias profesionales más adecuadas a su perfil (Saiz y Saiz, 1996, p. 380). Las pruebas específicas llevadas a cabo para medir las habilidades del sujeto consistían en el uso de diversos instrumentos, los cuales proporcionaban un valor de la fatiga, la inteligencia, el carácter, la emoción, la memoria y aptitudes especiales como la habilidad para la aritmética y para el dibujo. Pero no siempre había coincidencia entre los resultados y apreciaciones del enfoque científico y el procedente de las empresas (Soto, 1989, p. 242).

En general, los resultados de la política que pretendía vincular la escuela y los oficios no fueron brillantes. Entre las razones ofrecidas destaca la desconfianza de los trabajadores hacia las iniciativas calificadas como burguesas puestas en práctica por la Mancomunitat. En particular, se asociaban los procedimientos del IOP con las ideas relativas a la racionalización del trabajo y a una organización industrial científica, resultado del desmantelamiento del taller y de la aparición de la producción en serie (Iruela, 1993, p. 58).

A través del sistema de pensiones, otros profesionales relacionados con la enseñanza (inspectores y maestros) ampliaron sus conocimientos en las áreas cubiertas por la psicotecnia. Por mencionar unos nombres, en esta circunstancia se encontraron Rodolfo Tomás Samper, José del Peso Sevillano y Salvador Ferrer y Culubret, cuyas tareas como pensionados en Francia, Bélgica, Suiza y Alemania representan una buena muestra de ese interés. Así, en 1924, Rodolfo Tomás, maestro, se dedicó, como revela el índice de los trabajos y estudios llevados a cabo ${ }^{22}$, a los sistemas de ocupación profesional. Visitó escuelas, observó las metodologías de enseñanza profesional y participó en demostraciones y experimentos psicométricos realizados en laboratorios. A su vuelta, inició un ensayo de orientación profesional con cerca de un centenar de alumnos en el Colegio y Talleres de Nuestra Señora de la Paloma (Madrid). Por su parte, José del Peso ${ }^{23}$, recién nombrado Inspector de Primera Enseñanza, centró casi todos sus trabajos en el "Cabinet" de Orientación Profesional del admirado Instituto J. J. Rousseau de Ginebra. Entre los trabajos enviados a la Junta, llama la atención el que lleva por título "Diferenciación psicológica de individuos de ambos sexos"24. Comenta en las líneas previas a la presentación de los resultados que:

Las observaciones realizadas por mí entre alumnos adultos de Centros de enseñanza que he frecuentado, y entre alumnos de las escuelas primarias, han llevado a mi ánimo la convicción de que existen profundas diferencias, no solo cualitativas sino también cuantitativas, entre las distintas manifestaciones espiritua- 
les de hombre y de la mujer. Pero esta convicción no ha tenido otra base que la simple observación o intuición de los hechos o fenómenos que en torno a mí se han producido, lo cual hace que, a pesar de todo, no le conceda objetivamente otro valor que uno puramente provisional.

Mis ideas sobre el problema, demasiado apriorísticamente formado han sido últimamente reforzadas por las experiencias que, para resolver otros, he realizado o he visto realizar en los Establecimientos docentes que en Ginebra frecuento.

He querido hallarles una comprobación rigurosamente científica. A ello me han movido no solamente las inquietudes personales que han despertado en mí las cuestiones psicológicas, sino cuestiones de orden político y social:

De día en día el feminismo en todos sus matices se desarrolla más en los pueblos, y este desarrollo va imprimiendo nuevos rumbos a las sociedades y modificando ostensiblemente su estructura material y espiritual.

Las opiniones sobre su influencia actual y futura en el dinamismo social son tan discordantes como apasionadamente defendidas. Se han vertido raudales de tinta en elogios y en ataques. Pero la inmensa mayoría de las palabras que tal problema ha provocado han salido del corazón y no de la cabeza. La ciencia, que es la que únicamente puede esclarecerlo y orientarlo, no ha dicho aún la suya. $Y$ yo tengo un gran interés en conocerla. Por tal razón he afrontado el trabajo que menciono en estas cuartillas.

Más tarde reclama la autoridad de "dos de las figuras más ilustres de las ciencias psico-fisiológicas: M. Claparède y M. Lipmann (sic)", quienes supuestamente le apoyan en sus investigaciones (ellos mismos han confeccionado sendos tests para estimar los matices que el sentido de lo cómico reviste en ambos sexos). Añade finalmente que el trabajo no está sino en su fase inicial y que se espera que culmine en el futuro. En los ensayos ya realizados, una de las experiencias consistió en la determinación del tiempo de respuesta en la lectura, en la denominación de objetos y en la asociación de ideas. Se ofrecían a continuación los datos dispuestos en columnas. Pero los resultados no permitieron, como indica Del Peso, aventurar conclusión alguna, debido según el autor al escaso rigor científico de las experiencias: los aparatos carecían de fiabilidad y no existía una interpretación clara sobre la respuesta de los sujetos. Tampoco algunos designados por la Junta otorgaron una excesiva relevancia al conjunto de sus dedicaciones, a juzgar por la nota que escribió Martín Navarro ${ }^{25}$ el 28 de marzo de $1926^{26}$.
El tercero de los mencionados anteriormente, Salvador Ferrer, también Inspector de Primera Enseñanza, disfrutó de la pensión entre 1932 y 1933, después de dos intentos anteriores. Sus intereses, como los ya citados estaban centrados en la orientación profesional, la psicología experimental y las técnicas del trabajo. Estuvo vinculado al Instituto de Orientación Profesional de Barcelona, hecho que se refleja en la memora enviada a la Junta y que lleva por título "Contribución de la Escuela primaria a la orientación profesional" (Madrid, 1926). Allí hace una defensa del centro catalán y expone algunos de los problemas que en esos momentos preocupaban a los investigadores: la multiplicación de tests o la falta de un test general para medir la inteligencia ${ }^{27}$. Para paliar esta dificultad, en el Instituto se había creado, como asegura, la "Hoja psicológica", muy apreciada en ambientes académicos. Si Del Peso había concebido la esperanza de resolver algunos dilemas culturales a través de la tecnología vinculada a la psicología experimental, Ferrer no es menos ambicioso al proponer su "Estudio biológico y psicológico del niño", ponencia que presentó en la Asamblea de inspectores ${ }^{28}$. Allí explicaba el propósito último de este trabajo: "[ ] obtener conclusiones que sirvan para un fin utilitario en la máquina de la enseñanza nacional -es preciso que la Inspección de Primera Enseñanza se revista de un matiz técnico de que hoy carece". Se defiende frente a quien quiera presentarlo como un fanático de los números o bien un reduccionista que separa las diferentes actividades del sujeto o contempla a éste de forma aislada. No es esta, según insiste, su pretensión ni los números son la panacea, sin embargo sin ellos no obtendremos una "noción exacta del niño". "Todas las demás expresiones no son despreciables, ni mucho menos, pero carecen de importancia fundamental, entre otras razones porque al ser empíricas dependen del momento psíquico de quien las emplea" $Y$ esto quiere decir que para Salvador Ferrer los estudios deben consistir básicamente en "Medir [ ] Porque la resultante de una medida dará siempre un dato concreto, controlable, dispuesto a ser comparado con otro dato". Una tarea que remite a examinar la idoneidad de los tests, a determinar qué es exactamente lo que hay que medir y a establecer qué es un "niño normal"29.

\section{URBANISMO Y UTOPÍA SOCIAL}

Desde la extensión de la revolución industrial, particularmente en Inglaterra, los intelectuales o intelectuales-empresarios advirtieron que la tecnología arquitectónica podía emplearse para aliviar la condición desfavorable de amplios colectivos. Varios autores ya clásicos, como J. Bentham, W. Strutt y R. Owen hicieron aportaciones con la intención señalada (Pacey, 1980, pp. 237-250). Siguiendo esta perspectiva, en España se intentaron llevar a cabo algunos proyectos 
que tenían un inconfundible componente utópico, basados algunos de ellos en la idea de la "ciudad-jardín" del socialista inglés Ebenezer Howard (Arias, 2003, pp. 53-57). Centrándonos en el siglo XX, aunque con antecedentes en el anterior ${ }^{30}$, uno de los planes más destacados en el sentido mencionado fue el de la Ciudad Lineal de Arturo Soria. En realidad, la primera piedra se puso en 1894 y comprendía un eje de 50 $\mathrm{km}$. por $500 \mathrm{~m}$, con una sola calle central de $40 \mathrm{~m}$. de anchura y calles perpendiculares. Todas las avenidas debían tener edificios bajos rodeados de árboles, con una huerta en la parte posterior y un jardín en la entrada. Para evitar la impresión de "gueto", destinado a un barrio obrero, se diseñó con un propósito interclasista, también pensado para ubicar viviendas para personas con mayor poder adquisitivo y con servicios compartidos. Otro de los movimientos con componentes próximos al socialismo que pretendió cambiar el sistema de acceso a las viviendas y la planificación urbana fue el autodenominado GATEPAC (Grupo de Arquitectos y Técnicos Españoles para el progreso de la Arquitectura Contemporánea, creado en 1930), interesado en el desarrollo de la arquitectura racionalista y el urbanismo funcionalista e higienista ${ }^{31}$. Aquí prestaremos atención a las ideas y actuaciones de dos pensionados, Rafael Campalans y Julián Besteiro, cuyos planteamientos mantienen algunos puntos comunes con las concepciones vistas, en particular con los presupuestos de la estética utilitarista.

Rafael Campalans, ingeniero, pensador catalanista y militante socialista mantenía que con obreros ignorantes se alcanzaría la dictadura de un partido único, no una democracia social real ${ }^{32}$. Junto a esta preocupación por la formación de los trabajadores, se interesó por la dimensión social de la arquitectura y el urbanismo. En 1911 conseguía una beca concedida por la JAE para ampliar conocimientos de arquitectura industrial ${ }^{33}$. El plan comprendía, según declara, relacionarse con ingenieros y arquitectos de Francia y Alemania en la materia mencionada; examinar las obras más notables de ambos países y asistir a cursos (particularmente los del prestigioso Lucien Magne, de la Escuela Nacional de Bellas Artes). Estuvo disfrutando de la pensión 16 meses, ya que la solicitud inicial de un año fue prorrogada. La primera etapa la dedicó básicamente a la asistencia a cursos y durante la segunda, junto a estas tareas, trabajó en talleres de construcción y también visitó barrios industriales y obreros, escuelas técnicas, obras urbanas, centrales de energía, museos sociales y ciudades obreras. Aparte de estas alusiones poco precisas, poco más se conoce de sus viajes. Se sabe que también estuvo en Austria, Bélgica, Suiza, Holanda e Inglaterra. En París conoció a Jean Jaurés, diputado socialista en 1893, defensor de las causas obreras, pacifista y laicista, asesinado en las vísperas de la Primera Guerra Mundial. Posteriormente realizó una pequeña estancia en Bél- gica y se trasladó, asesorado por Jaurés, a Alemania, donde entró en contacto con la socialdemocracia y se interesó por la tecnología de ese país (Balcells, 1985, p.10). Finalizó en febrero de 1913, con 26 años, y un año después fue nombrado técnico de la Mancomunitat. Atendiendo de igual manera a las prioridades de la administración regional de Cataluña, fue designado director de la Escuela de Trabajo. Unos puestos que compatibilizó con cargos en la industria privada.

Su visión de Barcelona en esos años es representativa de su pensamiento. En un texto publicado en Revista Nova habla en contra de los planteamientos que la Exposición de Industrias Eléctricas, aún en proyecto ${ }^{34}$, ha alentado (Campalans, bajo el pseudónimo de Jaume deFrontanyà, 1914, p. 12). Destaca aquí su predisposición a la racionalización y ordenación del espacio urbano, idea que recuerda a la utopía foureriana y el falansterio como unidad arquitectónica y de convivencia, así como a los proyectos de las ciudades ideales. Se queja en ese breve escrito de las individualidades y las genialidades, cuya obsesión es la construcción de arcos y puentes colgantes; de la falta de una ordenación general de la ciudad; del descuido de calles y barrios; de la inexistencia de barrios-jardines donde "pueda vivir dignamente el pueblo que trabaja". E igualmente reclama la constitución de una Junta de la Gran Barcelona análoga a la Verband Gross-Berlin, la urbe alemana en la que probablemente esté pensando cuando se refiere a la conveniencia de fijarse en las ordenanzas municipales de una "ciudad civilizada".

En otros artículos dedicados particularmente a la estética arquitectónica revela algunos rasgos más de su mentalidad en esos años. En "Problemas de estética contemporánea" (Campalans, 1913, pp. 617-618), publicado con anterioridad a "Barcelona, alma mater", se presentan los presupuestos que guían su actitud hacia estas expresiones de la tecnología. Aquí ya rechaza, distanciándose de romanticismos y modernismos, las singularidades, así como la pretensión de confundir la arquitectura con un arte puro. La ciudad moderna para él, lejos de ofrecer una imagen de unidad, es una amalgama de estilos y caprichos. Cuando señala algunos ejemplos, como Notre Dame, el autor quiere hacer entender que estas construcciones son expresiones de una cultura, creaciones de un "alma colectiva". Así, en el arquitecto-ingeniero, que no artista, deben prevalecer criterios de utilidad, racionalidad y -añadiríamosde sociabilidad. En "D’Arquitectua. Entorn a l'evolució de l'istil" (Campalans, 1915, pp. 14-15) apunta una concepción del progreso que contribuye a entender los temas examinados hasta ahora y a relacionar su visión con idearios socialistas. El progreso humano, según Campalans, depende de la evolución social y de las producciones materiales, no es el resultado del perfeccionamiento del espíritu. La conclusión del autor es que la arquitectura es arte aplicado y que esta acepción 
no significa una merma de su dignidad y nobleza: en la utilidad hay una cierta belleza.

Las preocupaciones de otro socialista, Julián Besteiro, beneficiado igualmente por la política de pensiones de la JAE, se inscribe en esta atención a la relación entre urbanismo y reforma social. En 1909 viajaba a Alemania con la intención de trabajar en la Universidad de Múnich sobre el "Método de investigación de la Ética Científica, especialmente bajo la dirección del profesor [Theodor] Lipps". La estancia se prolongó hasta junio de 1911. Tras la solicitud de un permiso dirigido a la JAE para dedicar un semestre de permanencia en Leipzig y Berlín, el 11 de noviembre de 1909 se establecía en esta última ciudad, en este caso para trabajar con P. Bath. Más adelante, consiguió la consideración de pensionado para estudiar lógica en París y en Berlín durante el verano de 1913. Durante sus viajes no solo atendió a cuestiones académicas, sino que prestó atención a la realidad social y a los intensos debates existentes en esos momentos entre los representantes más notables de los movimientos obreros, adhiriéndose a la corriente de Karl Kautsky. Como confesaba años más tarde en una entrevista concedida a la revista La Esfera (14 de diciembre de 1918), la experiencia de la estancia en Alemania fue fundamental para consolidar sus convicciones socialistas. Durante la Dictadura de Primo de Rivera, además de su actividad política, Besteiro obtuvo nuevamente una pensión para realizar estudios en Inglaterra sobre la Worker's Educational Asscociation. En la solicitud hacía ver que los conflictos económicos y sociales de la época demandaban soluciones en las que había que contemplar no solo las respuestas de la filosofía teórica representada por la lógica, sino otros factores relativos a las transformaciones sociales ${ }^{35}$. Llegó a Londres el 25 de febrero de 1924 y allí se interesó por las políticas laboristas (en enero se había formado el primer gobierno laborista de la historia de Inglaterra), en especial por las medidas para facilitar el acceso a la vivienda de la población y por el funcionamiento de los sindicatos. También se preocupó por los planes relativos a la higiene pública y, sobre todo, por las relaciones entre la Universidad y el mundo obrero. En la conferencia pronunciada en la Casa del Pueblo de Madrid unos años antes, en 1920, ya había mostrado los principios de su ideario en los temas relativos al urbanismo, en los que se vinculaba la política de viviendas a la política social (Besteiro, $1920)^{36}$. En esas ideas se observa claramente la influencia del pensamiento de Kautsky (Sambricio, 1976 y 2003 , pp.12 y 13 y 18-19). Posteriormente, en plena guerra civil se creó el Comité de Reforma, Reconstrucción y Saneamiento de Madrid, presidido por Besteiro, que entre otros trabajos elaboró un Plan Regional para la capital. En su orientación, según se ha afirmado, influyeron los conocimientos de Fernando García Mercadal, representante del racionalismo arquitectónico e impulsor de la fundación de GATEPAC, la personalidad del propio Besteiro y su vinculación al ámbito cultural inglés (Terán, 1978, pp. 91-94). Además de las medidas para revitalizar el deterioro de los barrios, estaba prevista la creación de zonas de recreo y esparcimiento popular (las llamadas Playas del Jarama) mediante el aprovechamiento de las márgenes de los ríos con playas artificiales y edificaciones de uso colectivo.

\section{CONCLUSIONES}

Hemos comprobado que, partiendo de la idea de Sanford que se exponía al principio, varios expertos, representantes de la cultura española y alentados por la política científica de la JAE, optaron por buscar en qué condiciones se contemplaba la pertenencia de las personas a las máquinas, actitud que no cuestionaba la imparable extensión de la civilización artefactual ni su conveniencia para satisfacer los deseos de las multitudes. Para ello la pedagogía debía dejar de ser un arte y vincularse a la psicología experimental, ligada a su vez a un conjunto de técnicas de medición y de elaboración de tests ${ }^{37}$. Unas prácticas que, en correspondencia con las variables que detectaban y medían los aparatos, representaban una naturalización de las capacidades y procesos humanos, contribuyendo igualmente a una taxonomización de las personas. De esta manera se clasificaban las diferentes habilidades del individuo, práctica derivada de la crecente especialización social y de la división del trabajo.

También se buscaron soluciones en la educación elemental e intermedia que facilitaran el reconocimiento y la dignificación de la práctica. Los pensionados con intereses en la formación técnica no solo pensaron que ésta era beneficiosa para la adaptación del trabajador a los cambios industriales, sino que de igual manera defendieron que se contribuía con su difusión a un cambio de mentalidad dentro de la sociedad: se eliminaban las actitudes clasistas y también las amenazas de una subversión social. Junto a los propósitos anteriores, se extendieron diversos valores, como los sistemas descentralizados, las tendencias antiintelectualistas, la eliminación de la distinción entre pensamiento y técnica, la enseñanza manual, el contacto con la naturaleza y las fórmulas de la escuela-taller, la escuela-jardín, la escuela-granja, etc. Ideas que se sustentaban en las supuestas inclinaciones naturales del niño y en el racionalismo utilitario de la ciencia (enseñar a utilizar lo que sé o aprender utilizando). Valores que podían convertirse en la base de una política reformista que aproximara sensibilidades liberales y socialistas. 
En cuanto a los presupuestos de la psicología ocupacional, como afirma Francisco Villacorta, si bien se intentaba mantener una distancia con el taylorismo, se reconocían las virtudes del "enfoque tecnocrático de los problemas del mundo social, en contraposición radical a los enfrentamientos entre clases y a los mecanismos clásicos de abordarlos por parte de las políticas públicas" (Villacorta, 2012, p. 160).

La adaptabilidad a la máquina se promovió de igual manera con la planificación de edificios y de entornos urbanos y naturales. Las multitudes podían disfrutar de viviendas a escala humana rodeadas de árboles y jardines y también de amplios espacios de recreo, destinados al "ocio de masas", como las piscinas, parques deportivos y playas artificiales. Ejemplo de esto último es la Playa del Jarama, cuyo proyecto buscaba atender a "los miles de almas que por los ferrocarriles de MZA y de Arganda, así como por los autobuses de Paracuellos, San Fernando y Mejorada salen de Madrid todos los domingos en busca del río Jarama que, por tener un cauce superior al del Manzanares, permite ser utilizado» (Sambricio, 2003, p. 60).

Para humanizar los enfoques puramente mecánicos de los problemas, que contemplaban solo tiempos y movimientos, en la metodología psicotécnica se introdujeron otras variables, como las motivaciones, intere-

\section{NOTAS}

1 Sobre este tema, Vicente Cacho Viu (1997) y Leoncio LópezOcón Cabrera (2003).

2 Partiendo de los análisis de M. Foucault (Foucault, 1988, pp. 49 y 50 ), se entiende aquí que en la tecnocracia la matriz de la razón práctica es la de la producción industrial.

3 Cabe incluir en esta orientación, dedicada a la integración social siguiendo los enfoques tecnocientíficos, el estudio de la educación de niños con problemas orgánicos y mentales, sin embargo en este trabajo, por las limitaciones de espacio, no se profundizará en este tema, sobre el que remitimos a Mariano Herraiz Gascuena (1995); de igual manera, se enmarcan en estas tendencias las corrientes higienistas, a las que, por las mismas razones, tampoco prestaremos atención, si bien sugerimos por su interés los trabajos de Rafael Huertas (2008) y Anna Quintanas (2011).

4 En particular durante la Dictadura de Primo de Rivera, si bien hay casos de años anteriores y también de la 2 a República.

5 Con posturas aún más radicales se manifestó Miguel de Unamuno, de quien citaremos, entre otros variados ejemplos, la distopía "Mecanópolis", publicada en 1913. ses, habilidades motoras, capacidad visual... Pero sus logros fueron desiguales, como los proyectos mencionados a lo largo del texto, ya que como ocurre en los procedimientos ajustados a una epistemología tecnocientífica, las proyecciones y correlaciones solo pueden efectuarse a partir de elementos manipulables, medibles y detectables por los aparatos. Estos definen condiciones muy generales, que no alcanzan a prever las situaciones específicas en marcos reales. En la motivación y ánimo de los trabajadores, probablemente en su efectividad y dedicación, también influían otros factores culturales y relativos a las políticas empresariales y sociales no contemplados directamente en los artefactos, como las remuneraciones, las posibilidades de promoción, la regulación de los horarios, la formación, la seguridad y otras demandas laborales.

\section{AGRADECIMIENTOS}

Trabajo realizado en el marco del proyecto de Investigación "Educación 'integral' para los jóvenes bachilleres: cambios promovidos por la JAE en la enseñanza secundaria (1907-1936)". Plan Nacional de I+D+I (2008-2011) (HAR 2011-28368). Deseo mostrar mi agradecimiento por sus valiosos comentarios a las versiones previas de este trabajo a Leoncio López-Ocón, Francisco Villacorta, Leonor González y José Manuel Azcona.
6 En esa primera etapa se enmarcan además los trabajos de Ramiro Suárez Bemúdez y Amadeo Pontes Lillo. El primero era catedrático de Electroquímica y en el momento de la solicitud dirigida a la JAE era director de la Escuela Central Superior de Artes Industriales; durante los meses que permaneció en el extranjero se interesó por la organización de las escuelas primarias, los talleres de trabajo manual, los materiales de enseñanza, las escuelas municipales, escuelas de artes y oficios, etc. Pontes Lillo, ingeniero industrial y dedicado profesionalmente a la ingeniería, fue becado en 1913 y sus preocupaciones se centran en los muesos industriales y en el tema de la formación profesional de la mujer (Marín, 1991, p. 339 y pp. 281-282).

7 Más tarde menciona otros ejemplos respetables, en los que se "rinde culto a la directa observación y personal experiencia", como el de Bedales School (Petersfield, Inglaterra); en este centro "niños y niñas se ejercitan en el taller de carpintería, en cocinar, en coser, en jardinería, en los menesteres todos de una casa de labor (ordeñar la leche, obtener la crema, hacer queso, manteca, cuidar animales de corral, colmenas, obtener la miel, la cera, etc.), y los muchachos, además, en el taller de metales" (Miralles, 1911, p. 215).

8 Véase sobre la inserción de la tecnología en este tipo de centros, Víctor Guijarro y Leonor González (2013). 
9 De estos dos pensionados hablaremos en el siguiente apartado, el dedicado a la psicotecnia.

10 Luis Castellá era profesor de Aritmética y Geometría prácticas en la Escuela Industrial de Cartagena en 1921 y estaba interesado en los métodos de enseñanza práctica y en la orientación profesional; Gervasio Manrique era inspector de primera enseñanza en Soria y estaba interesado en la pedagogía social y en la orientación profesional, así como en la aplicación de innovaciones en la Escuela de Artes y Oficios de la ciudad citada; Leonor Serrano, inspectora de enseñanza y abogada, escribió, entre otras obras, La enseñanza complementaria obrera (1926) y La educación y las profesiones femeninas, como complemento de La educación de la mujer de mañana y El Método Montessori (1928) (Marín, 1999, pp. 73-75, 224-225 y 331-333).

11 Estrecho colaborador de César de Madariaga, figura central en la gestión de la Junta de Pensiones de Ingenieros y Obreros, que promovió un ideal humanista en su enfoque de la organización científica del trabajo; Valerí, por su parte, licenciado en Derecho y en Filosofía y Letras, era en 1925-26 vicesecretario de la Secretaría de Enseñanza Técnica del Ministerio de Trabajo y probablemente trasladó sus experiencias a los proyectos educativos que estaban teniendo lugar en la Universidad de Barcelona (Villacorta, 2012, pp 201 y 649 y ss.).

12 Gaceta de Madrid, 5 de noviembre de 1924, no 310

13 Expediente JAE/146-27, solicitud de 23 de febrero de 1925.

14 Expediente JAE/146-27, solicitud de 23 de febrero de 1925 . La cursiva es del autor.

15 Sobre la respuesta de las ciencias humanas a los cambios y, en particular, sobre la psicología aplicada, Roger Smith (1997, pp. 606-625).

16 Anteriormente había sido catedrático de la misma disciplina en los institutos de Orense y Toledo; sobre su trayectoria profesional e intelectual, Florentino Blanco, Jorge Castro y Raquel de Castro (1996) y Natividad Araque y Carmen Rodríguez (2011).

17 Expediente JAE/ 89-384, 1 de octubre de 1910.

18 Este tema forma parte de un trabajo de investigación que en estos momentos está en fase de desarrollo y que será presentado en el Congreso de la Sociedad Española de Historia de la Ciencia del año 2014.

19 Sobre su trayectoria, Dolores Saiz y Milagros Saiz (1996) y Luis Miguel Iruela Cuadrado (1993).

20 Luis Miguel Iruela mantiene que Campalans, del que hablaremos más adelante, ejerció una importante influencia en las ideas políticas de Emilio Mira (Iruela, 1993, p. 57).

21 El Primer Congreso Internacional de Psicotecnia de 1920, que tuvo lugar en Ginebra, fue organizado por Edouard Claparède, director del Instituto J. J. Rousseau, y contó con la participación de Decroly y Bovet. Como representantes del IOP acudieron Ruiz Castella y Emilio Mira.

22 Rodolfo Tomás, "Índice de los trabajos y estudios llevados a cabo durante los dos meses de pensión, comprendidos entre el
29 de mayo y el 28 de julio últimos, formando parte de un grupo de maestros", Expediente JAE/142-90.

23 Obtuvo dos pensiones, en 1924 y en 1925

24 Incluido en la carpeta "Datos sobre algunos de los trabajos científicos realizados por José del Peso, Inspector de Enseñanza pensionado en Ginebra por la Junta de Ampliación de Estudios", 1926, Archivo JAE/ P-60.

25 Martín Navarro Flores, 1871-1950, fue profesor de la Institución Libre de Enseñanza y del Instituto-Escuela de Madrid, y también catedrático de Psicología en el Instituto de Tarragona, donde publicó en 1914 un interesante Manual de Psicología Experimental, el primero de estas características que apareció en España.

26 Expediente JAE/115-391.

27 "Una de las dificultades en que tropieza la Psicología Experimental es el número considerable de tests existentes para el establecimiento de baremos de utilidad pública. Ello obliga a un continuo trabajo de contrastación que perjudica la intensidad con que podrían llevarse a cabo las valoraciones", Salvador Ferrer, "Contribuciones de la Escuela Pública", Madrid, 1926, Archivo JAE/F-33.

28 Enviada a la Junta en su petición de 1931

29 Salvador Ferrer, "Estudio biológico y psicológico del niño", Archivo JAE/F-34.

30 En el siglo XIX, destaca la propuesta de Ángel Fernández de los Ríos El Futuro Madrid de 1868, destinada a resolver el problema del alojamiento obrero; en su proyecto estaba prevista la construcción de cuatro barrios, que llevarían los nombres de inspiración saintsimoniana "Trabajo", "Economía", "Cooperación" e "Instrucción"; estos ideales los recuperará la Cooperativa de Casas Baratas “Pablo Iglesias” (Arias, 2003, p. 53).

31 Tuvo su foco de actuación principalmente en Barcelona, con el apoyo de la Generalitat.

32 Sobre la trayectoria intelectual y política de Campalans, Albert Balcells (1985) y Santiago Riera i Tuèbols (1979).

33 En el expediente de la JAE correspondiente a su solicitud (JAE/28-128) señala que ha terminado la carrera de Ingeniero Industrial según el plan de la Facultad de Ciencias de Barcelona (enseñanza oficial) y el de la Escuela de Ingenieros Industriales de Barcelona (enseñanza no oficial)

34 Finalmente no llegó a celebrarse por el conflicto bélico; en realidad se convirtió en el embrión de la Exposición Internacional de 1929.

35 Archivo JAE/20-323.

36 Julián Besteiro, “Conferencia dada en la Casa del Pueblo sobre el problema de la vivienda", Madrid, 1920

37 Esta pretensión la defendieron también otras figuras con una relación estrecha tanto con la Institución Libre de Enseñanza como con la Junta, como Julián Besteiro y Domingo Barnés. 


\section{BIBLIOGRAFÍA}

André, Eloy Luis (1912), "El porvenir de la Filosofía científica en España e Hispano-América", Nuestro Tiempo, XII, 159, Madrid, pp. 309-328.

André, Eloy Luis (1931), Psicología experimental, 4ạ ed. Madrid, Imp. Sáez Hermanos.

Arias González, Luis (2003), Socialismo y vivienda obrera en España (1926-1939), Salamanca, Ediciones Universidad de Salamanca.

Balcells, Albert (1985), Rafael Campalans, socialismo català. Biografia i textos, Barcelona, Publicacions de l'Abadia de Montserrat.

Ballesteros Usano, Antonio (1925), “Características de la enseñanza primaria en Francia, Bélgica y el Cantón Suizo de Neuchâtel”, Anales de la JAE, t. XIX.

Blanco, Florentino; Jorge Castro y Raquel de Castro (1996), "Eloy Luis André (1876-1935)". En: Milagros Saiz y Dolores Saiz (coords.), Personajes para una historia de la psicología en España, Madrid, Pirámide, pp. 253-267.

Cacho Viu, Vicente (1997), Repensar el noventa y ocho, Madrid, Biblioteca Nueva.

Campalans, Rafael (1913), "Problemas de estética contemporánea", Hispania: Política, Comercio, Literatura, Artes y Ciencias, vol. 2, 18 (1), pp. 617-618.

Campalans, Rafael (1915), “D’Arquitectua. Entorn a l'evolució de I'istil', La Revista: quaderns de publicació quincenal, 1, pp. 14-15.

Esquirol, Josep M. (2001), La filosofía contemporánea y la técnica. De Ortega a Sloterdijk, Barcelona, Gedisa.

Foucault, Michel (1988), Tecnologías del yo, Barcelona-Buenos Aires-México, Ediciones Paidós Ibérica/ICE Universidad Autónoma de Barcelona.

Frontanyá, Jaume de (1914), "Barcelona 'alma mater'”, Revista Nova, 1, p. 12.

Guijarro, Víctor y Leonor González (2013), “La presencia de la tecnología en la utopía pedagógica: el Instituto de San Isidro y los Institutos-Escuela promovidos por la JAE". En: Leonor González y Vicente J. Fernández (eds.), El Instituto de San Isidro. Saber y patrimonio: apuntes para una historia, Madrid, CSIC, pp. 173-197.

Herraiz Gascuen, Mariano (1995), Una aproximación a la educación especial española del primer tercio del siglo XX, Cuenca, UCLM.

Huertas, Rafael (2008), Los laboratorios de la norma. Medicina y regulación social en el estado liberal, Barcelona, Octaedro-CSIC.

Irigoyen, E. (2002), "El arte es una máquina de (des)montaje. Fordismo-taylorismo y vanguardias artísticas a principios del siglo XX", Scripta Nova, Revista Electrónica de Geografía y Ciencias Sociales, Universidad de Barcelona, vol. VI, 119 (7), [en línea], disponible en: http://www.ub.es/geocrit/sn/sn119-7.htm [consultado el 5/8/2013]
Iruela Cuadrado, Luis Miguel (1993), Psiquiatría, psicología y armonía social: La vida y la obra de Emilio Mira y López, Barcelona, Universidad de Barcelona.

Junta para la Ampliación de Estudios (1927), Memoria correspondiente a los cursos 1924-1925 y 1925-1926, Madrid.

López-Ocón Cabrera, Leoncio (2003), Breve historia de la ciencia española, Madrid, Alianza.

Marín Eced, Teresa (1988), "Influencias europeas en la formación profesional de docentes españoles durante la II República Española", Revista de Educación, 285, pp. 93-110.

Marín Eced, Teresa (1991), Innovadores de la educación en España: becarios de la Junta para la Ampliación de Estudios, Cuenca, Servicio de Publicaciones de Castilla-La Mancha.

Miralles Solbes, Lorenzo (1911), “Estudio crítico de los métodos para la enseñanza de las primeras nociones de las ciencias experimentales en las Escuelas", Anales de la JAE, III, Memoria 4a , pp. 146-226.

Mülberger, Annette (2014), "Encuentro con la psicología alemana", Mente y cerebro, 64, pp. 89-92.

Münsterberg, Hugo (1913), Psychology and Industrial Efficiency. Boston y Nueva York.

Natividad Araque y Carmen Rodríguez (2011), “Cien años de enseñanza de la Filosofía en el Instituto Cardenal Cisneros", Revista Complutense de Educación, 22 (1), pp. 47-67.

Ortega y Gasset, José (1908), "Asamblea para el progreso de las ciencias", El Imparcial, Madrid, 27 de julio.

Ortega y Gasset, José (2006), “Primitivismo y técnica”. En: Fundación Ortega y Gasset, Centro de Estudios Orteguianos (ed.), La rebelión de las masas, Obras completas, Madrid, Taurus, tomo V, pp. 564-567.

Ortega y Gasset, José, (2006), "El esfuerzo para ahorrar esfuerzo es esfuerzo-El problema del esfuerzo ahorrado-La vida inventada". En: Fundación Ortega y Gasset, Centro de Estudios Orteguianos (ed.), Obras completas, Madrid, Taurus, tomo V.

Pacey, A. (1980), El laberinto del ingenio. Ideas e idealismo en el desarrollo de la tecnología, Barcelona, Gustavo Gili, pp. 237-250.

Pannabecker, J. R. (1996), "Diderot, Rousseau, and the mechanical arts: Disciplines, systems, and social context", Journal of Industrial Teacher Education, 33 (4), pp. 6-22.

Quintanas, Anna (2011), “Higienismo y medicina social: poderes de normalización y formas de sujeción de las clases populares", ISEGORÍA. Revista de Filosofía Moral y Política, 44, pp. 274-284.

Riera i Tuèbols, Santiago (1979), "Rafael Campalans, enginyer i politic", L'Avenç, 16, pp. 6-11. 
Rodríguez Guerrero, Carmen (2012), "El laboratorio de psicología científica del Instituto del Cardenal Cisneros", Cabás, no7, [en línea], disponible en: http://revista.muesca.es [consultado el 21/10/2013]

Saiz, Dolores y Saiz, Milagros (1996), "Emilio Mira y la psicotecnia”. En: Milagros Saiz y Dolores Saiz (coords.), Personajes para una historia de la psicología en España, Madrid, Pirámide, pp. 375-398.

Sambricio, Carlos (1976), “Introducción”. En: Carlos Sambricio, Luis Lacasa: escritos 1918-1936, Madrid, Colegio Oficial de Arquitectos de Madrid, pp. 12 y 13.

Sambricio, Carlos (2003), “Introducción”. En: Julián Besteiro, Esquema y bases para el desarrollo del Plan Regional de Madrid (Madrid, 1939), Madrid, Consejería de Obras Públicas, Urbanismo y Transportes.

Sambricio, Carlos (2004), "Las Playas del Jarama, proyecto del GATEPAC, Grupo Centro", Actas del IV Congreso del DoCoMoMo
Ibérico, 6- 8 Nov 2003, Arquitectura moderna y turismo: 19251965, Valencia, Fundación DOCOMOMO Ibérico.

Sanford, Charles L. (1981). "Tecnología y cultura a finales del siglo XIX: la voluntad de poder". En: Melvin Kranzberg y Caroll W. Pursell, Jr. (eds.), Historia de la tecnología. La técnica en Occidente de la Prehistoria a 1900, vol. 2, Barcelona, Gustavo Gili.

Smith, Roger (1997), The Fontana History of the Human Sciences, Londres, Fontana Press.

Soto Carmona, Álvaro (1989), El trabajo industrial en la España Contemporánea, 1874-1936, Barcelona, Anthropos.

Terán, Fernando de (1978), Planeamiento urbano en la España contemporánea, 1900-1980, Madrid, Alianza.

Villacorta Baños, Francisco (2012), La regeneración técnica. La Junta de Pensiones de Ingenieros y Obreros en el Extranjero (19101936), Madrid, CSIC. 